\title{
Metformin suppresses the invasive ability of pancreatic cancer cells by blocking autocrine TGF- $\beta 1$ signaling
}

\author{
WANXING DUAN $^{1}$, WEIKUN QIAN $^{1}$, CANCAN ZHOU $^{1}$, JUNYU CAO $^{1}$, TAO QIN $^{1}$, YING XIAO $^{1}$, \\ LIANG CHENG $^{1}$, JIE LI $^{1}, \mathrm{KE} \mathrm{CHEN}^{1}$, XUQI LI ${ }^{2}$, JIGUANG MA ${ }^{3}$ and QINGYONG MA ${ }^{1}$ \\ Departments of ${ }^{1}$ Hepatobiliary Surgery, ${ }^{2}$ General Surgery and ${ }^{3}$ Anesthesiology, \\ The First Affiliated Hospital, Xi'an Jiaotong University, Xi'an, Shaanxi 710061, P.R. China
}

Received January 20, 2018; Accepted June 18, 2018

DOI: $10.3892 / o r .2018 .6518$

\begin{abstract}
Pancreatic ductal adenocarcinoma (PDAC) is a highly invasive neoplasm with a 5-year survival rate of $<8 \%$. Metformin, the most widely used antidiabetic drug in the world, has been shown to exert anticancer activities in epidemiological and animal studies. Our previous studies revealed that metformin suppressed desmoplasia in PDAC by reducing TGF- $\beta 1$ production in cancer cells. The aim of the present study was to investigate the effects of metformin on invasion and epithelial-mesenchymal transition (EMT) in pancreatic cancer and to reveal the underlying mechanisms. In the present study, we revealed that metformin suppressed migration, invasion and EMT changes in pancreatic cancer cells. Furthermore, metformin reduced TGF- $\beta 1$ production and $S m a d 2 / 3$ phosphorylation in pancreatic cancer cells. In addition, treatment with recombinant TGF- $\beta 1$ recovered the metformin-mediated invasion inhibition and EMT changes. Treatment with metformin also suppressed tumor growth, invasion and EMT in LSL-Kras ${ }^{G l 2 D /+}$, Trp $53^{f / /}$ and Pdxl-Cre (KPC) transgenic mice that harbor spontaneous pancreatic cancer. Collectively, our study revealed a new possible mechanism for the antitumor effects of metformin via autocrine TGF- $\beta 1 /$ Smad2/3 signaling in PDAC.
\end{abstract}

\section{Introduction}

Pancreatic ductal adenocarcinoma (PDAC) is one of the most aggressive forms of human pancreatic cancer. Although in-depth knowledge of the disease has been elucidated and an extensive amount of research has been conducted in the past few decades, the effectiveness of treatment for this malignancy

Correspondence to: Dr Qingyong Ma, Department of Hepatobiliary Surgery, The First Affiliated Hospital, Xi'an Jiaotong University, 277 West Yanta Road, Xi'an, Shaanxi 710061, P.R. China

E-mail: qyma56@xjtu.edu.cn

Key words: metformin, TGF- $\beta 1$, epithelial-mesenchymal transition, metastasis, pancreatic ductal adenocarcinoma, LSL-Kras ${ }^{G 12 D /+}$, Trp53 $3^{f l+}$, Pdxl-Cre (KPC) transgenic mice has not substantially improved. PDAC remains the fourth leading cause of cancer-related mortality in the United States, with a 5-year survival rate of $<8 \%$ (1). PDAC may go undetected until patients have progressed to an advanced stage. More than $80 \%$ of patients have already missed the opportunity for radical surgery at the time of initial diagnosis (2). Due to the aggressive nature of this malignancy and its non-sensitivity to radiotherapy and chemotherapy, local recurrence and distant metastasis after initial 'curative' resection is an unresolved clinical problem for PDAC patients (3).

Active invasion and metastasis have been recognized as hallmarks of cancer (4). Accumulating evidence has indicated that epithelial-mesenchymal transition (EMT), which initiates an invasion-metastasis cascade, is a feature of aggressive tumors $(5,6)$. During EMT, cancer cells disassemble their epithelial junctions and suppress the expression of junctional proteins. Consequently, the cancer cells acquire the abilities to invade, resist apoptosis and disseminate (7). E-cadherin, a component of adherent junctions, is lost during EMT and cancer progression. Conversely, another adherent junction protein, $\mathrm{N}$-cadherin is upregulated in cancer cells. A previous study revealed that cadherin switching was a main event in EMT and was necessary for the increased motility of cancer cells (8). Accumulating evidence has revealed that EMT plays a crucial role in the invasion and metastasis of pancreatic cancer $(9,10)$. Recently, several studies have revealed that many growth factors and cytokines, as well as cellular signaling pathways, could induce EMT. One such cytokine is transforming growth factor- $\beta 1$ (TGF- $\beta 1$ ) (11).

Compared with other treatments, metformin, the first-line treatment for managing type 2 diabetes mellitus, has been associated with reduced cancer burden in epidemiological studies in diabetic patients $(12,13)$. There is also evidence that metformin use is associated with a reduced risk of prostate (14), colon (15) and pancreatic cancer (16). Given the anticancer properties and the safety profile of metformin, this treatment has attracted great interest from cancer researchers worldwide (17). In the past, exciting preclinical studies have shown that metformin can inhibit cancer cell growth both in vitro and in vivo (18-20). Using the $L S L-K r a s G 12 D /+$, Trp53fl/+ and Pdxl-Cre (KPC) mouse models, our previous study indicated that metformin suppressed the initiation and progression of pancreatic cancer (21). In another study (22), our data revealed 
that metformin-mediated AMPK activation in cancer cells inhibited pancreatic cancer progression by suppressing the desmoplastic reaction in tumor tissues. Further mechanistic experiments revealed that metformin reduced TGF- $\beta 1$ production in cancer cells, thus suppressing the TGF- $\beta 1$-induced activation of pancreatic stellate cells (PSCs) (22). Based on these observations, we hypothesized that metformin could inhibit the invasion and metastasis of pancreatic cancer cells by blocking the autocrine TGF- $\beta 1$ signaling. In the present study, we used the KPC transgenic mouse model of pancreatic cancer to demonstrate that metformin inhibited the invasion and metastasis of pancreatic cancer by suppressing TGF- $\beta 1 /$ Smad $2 / 3$ signaling.

\section{Materials and methods}

Cell culture and reagents. Human PDAC cell lines Panc-1 and BxPC-3 were purchased from The Chinese Academy of Sciences Cell Bank of Type Culture Collection (CBTCCCAS; Shanghai, China). The cells were maintained in the appropriate medium (DMEM; HyClone Laboratories, Logan, UT, USA) for Panc-1 and RPMI-1640 medium for BxPC-3 (HyClone Laboratories) supplemented with $10 \%$ fetal bovine serum (FBS; HyClone Laboratories; GE Healthcare Life Sciences, Logan, UT, USA) and $1 \%$ penicillin/streptomycin in a humidified atmosphere containing $5 \% \mathrm{CO}_{2}$ at $37^{\circ} \mathrm{C}$. Primary antibodies for E-cadherin (1:1,000; cat. no. 3195), N-cadherin (1:800; cat. no. 13116), MMP-2 (1:1,000; cat. no. 40994) and vimentin (1:1,000; cat. no. 5741) were purchased from Cell Signaling Technology, Inc. (Danvers, MA, USA). Antibodies against $\beta$-actin $(1: 1,000$; cat. no. A5441) were obtained from Sigma-Aldrich; Merck (St. Louis, MO, USA). Primary antibodies for TGF- $\beta 1$ (1:800; cat. no. ab92486), Smad2/3 $(1: 1,000$; cat. no. ab202445) and p-Smad2/3 (1:1,000; cat. no. ab63399) were purchased from Abcam (Cambridge, MA, USA) and anti- $\alpha$-SMA (1:800; cat. no. A03744) was obtained from Boster Biological Technology (Wuhan, China). All secondary antibodies (goat anti-rabbit IgG-HRP (1:10,000; cat. no. ssc-2004) and goat anti-mouse IgG-HRP (1:10,000; cat. no. sc-2005) were purchased from Santa Cruz Biotechnology (Santa Cruz, CA, USA). Recombinant TGF- $\beta 1$ (T7039) was obtained from Sigma-Aldrich. Metformin (Sigma-Aldrich; Merck) was dissolved in phosphate-buffered saline (PBS) as a stock solution of $100 \mathrm{mM}$. Working dilutions of metformin were made in culture medium immediately before use.

Wound healing assays. Wound healing assays were conducted to assess the migration ability of cancer cells. Briefly, cancer cells were seeded in 6-well plates and when they almost covered the well, the cells were serum-starved and then treated with metformin $(2 \mathrm{mM})$ for $24 \mathrm{~h}$. Subsequently, the cells were scratched using a $200-\mathrm{ml}$ sterile pipette tip. Images of the matched-pair wound regions at 0 and $24 \mathrm{~h}$ were obtained at a magnification of $\mathrm{x} 100$ using a light microscope (Nikon Instruments, Inc., Tokyo, Japan).

Matrigel invasion assay. Matrigel invasion assays were performed to investigate the invasive ability of cancer cells. In brief, cancer cells were serum-starved overnight and then pretreated with or without metformin plus TGF- $\beta 1$ ( $2 \mathrm{ng} / \mathrm{ml}$ ) for $24 \mathrm{~h}$. Subsequently, the cells were trypsinized, and $5 \times 10^{4}$ cells and $200 \mu \mathrm{l}$ of serum-free medium were added to Matrigel-coated inserts (BD Biosciences, Franklin Lakes, NJ, USA). Complete medium (500 $\mu \mathrm{l})$ was added to the lower chamber. Following incubation for $48 \mathrm{~h}$, the invaded cells were stained with $0.1 \%$ crystal violet solution for $15 \mathrm{~min}$ at room temperature, and 10 randomly selected fields were photographed with a light microscope (Nikon Instruments, Inc.) at a magnification of $\times 200$.

Immunofluorescence analysis. Following the designated treatment, immunohistochemical analyses were performed according to our previously described protocol (23). Images were pseudocolored using a fluorescence microscope (Nikon Eclipse Ti-s; Nikon Instruments, Inc.) with the appropriate excitation and emission (blue, $440-450 \mathrm{~nm}$; green, $530-550 \mathrm{~nm}$; red, $630-650 \mathrm{~nm}$ ) spectra at a magnification of $\mathrm{x} 400$. Image-Pro Plus software (version 6.0; Media Cybernetics Inc., Rockville, MD, USA) was used for further analysis.

Western blotting. Total cellular protein was extracted using RIPA lysis buffer (Beyotime Institute of Biotechnology, Guangzhou, China). Protein concentrations were analyzed with a BCA protein assay kit (Beyotime Institute of Biotechnology) according to the manufacturer's instructions. The same amounts of protein $(150 \mu \mathrm{g})$ from the samples were separated by $10 \%$ SDS-PAGE and then transferred onto polyvinylidene difluoride (PVDF) membranes as previously described (24). The membranes were blocked with $5 \%$ non-fat dry milk in TBST [10 mM Tris- $\mathrm{HCl}$ (pH 8.0), $150 \mathrm{mM} \mathrm{NaCl}, 0.05 \%$ Tween-20]. Then membranes were incubated with primary antibodies overnight at $4^{\circ} \mathrm{C}$. After three washes of $10 \mathrm{~min}$ each in TBST, the membranes were incubated with HRP-conjugated secondary antibodies for $1 \mathrm{~h}$ and subsequently washed again. Protein expression was visualized using enhanced chemiluminescence (EMD Millipore, Billerica, MA, USA) and the ChemiDoc XRS imaging system (Bio-Rad Laboratories, Inc., Hercules, CA, USA). $\beta$-actin was used to ensure equivalent protein loading.

In vivo study. All experimental protocols were approved by the Ethical Committee of The First Affiliated Hospital of Medical College, Xi'an Jiaotong University (Xi'an, China). LSL-Kras ${ }^{G l 2 D /+}$; Trp $53^{f /+}$; Pdxl-Cre (KPC) mice were generated and maintained as previously described (21). A total of 20, 6-week-old mice (female; average weight, $20 \pm 1.79 \mathrm{~g}$ ) were divided into two groups of 10 mice each. Mice in the metformin group were gavaged with vehicle (sterile water) or metformin $(200 \mathrm{mg} / \mathrm{kg})$ daily for 4 weeks. All mice were housed under pathogen-free conditions with a 12:12-h dark/light cycle under constant temperature $\left(24^{\circ} \mathrm{C}\right)$. At the termination of the experiment, the mice were euthanized by $30 \% \mathrm{CO}_{2}$ asphyxiation, followed by cervical dislocation. Tissues were harvested and fixed in formalin. Tumor samples were prepared for further histological analysis.

Statistical analysis. Each experiment was performed at least three times. All quantitative data were analyzed using SPSS version 15.0 (SPSS, Inc., Chicago, IL, USA) and are expressed 
$\mathbf{A}$

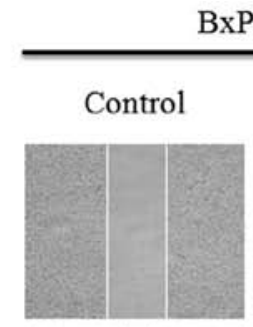

$24 \mathrm{~h}$

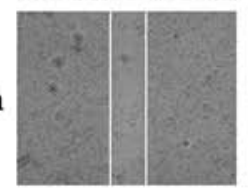

BxPC-3
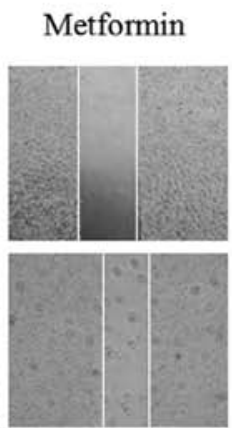
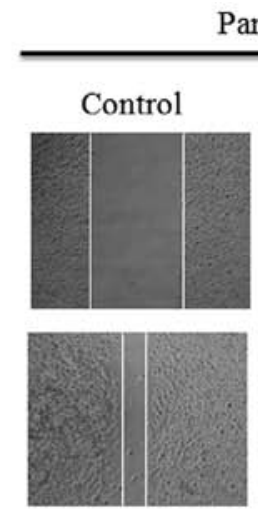

Panc-1
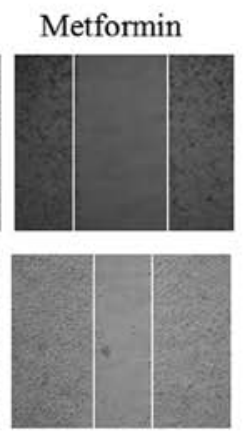

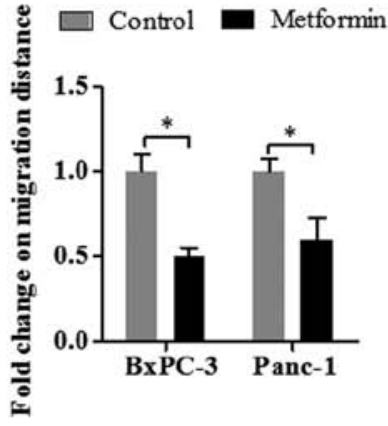

BxPC-3

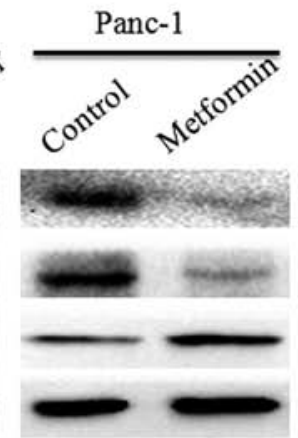

Figure 1. Metformin inhibits the migration and invasion of pancreatic cancer cells. (A) Wound healing assays were performed to assess the migration ability of Panc-1 and BxPC-3 cells following $2 \mathrm{mM}$ metformin treatment. Images were acquired at 0 and $24 \mathrm{~h}$ at a magnification of $\mathrm{x} 100$; ${ }^{*} \mathrm{P}<0.05$. (B) Panc-1 and BxPC-3 cells were treated with $2 \mathrm{mM}$ metformin for $24 \mathrm{~h}$. Cell invasion ability was evaluated by Matrigel invasion assays. Error bars display the mean \pm SD of three independent experiments. ${ }^{*} \mathrm{P}<0.05$, magnification, $\mathrm{x} 200$. (C) Western blot assays were performed to evaluate the effects of metformin on the EMT markers E-cadherin and N-cadherin and the invasion-related molecule MMP-2.

as the mean \pm standard deviation (SD). Two-tailed unpaired Student's t-tests were used to analyze the data between groups. $\mathrm{P}<0.05$ was considered to indicate a statistically significant difference.

\section{Results}

Metformin attenuates the migration and invasion abilities of pancreatic cancer cells. First, the effect of metformin on the migration ability of pancreatic cancer cells was assessed by wound healing assays. Similarly, with our previous study, $2 \mathrm{mM}$ metformin was also used in the present study (22). As displayed in Fig. 1A, the migration ability of pancreatic cancer cells was significantly suppressed by $2 \mathrm{mM}$ metformin treatment. To investigate the effect of metformin on the invasion ability of pancreatic cancer cells, Matrigel invasion assays were performed. The results revealed that the invasion ability of pancreatic cancer cells was decreased by $2 \mathrm{mM}$ metformin treatment (Fig. 1B). These results indicated that metformin inhibited the invasion and migration capacities of pancreatic cancer cells in vitro. Available evidence has indicated that active EMT in cancer cells enhances their invasion and metastasis abilities. To explore whether metformin has an effect on EMT-related molecules, the protein expression levels of E-cadherin, N-cadherin and MMP-2 were detected in pancreatic cancer cells following exposure to $2 \mathrm{mM}$ metformin for $48 \mathrm{~h}$. The western blotting results revealed that metformin treatment decreased the protein expression levels of N-cadherin and MMP-2 and increased the protein expression levels of E-cadherin in both BxPC-3 and Panc-1 cells (Fig. 1C).

Metforminsuppresses TGF- $\beta 1 /$ Smad2/3 signaling inpancreatic cancer cells. Canonical TGF- $\beta 1 / \mathrm{Smad} 2 / 3$ signaling has been revealed to play an important role in EMT in various epithelial cells (11). Our previous study (22) revealed that metformin inhibited TGF- $\beta 1$ production in pancreatic cancer cells by inducing AMPK activation. To further explore the effects of metformin on TGF- $\beta 1 / \mathrm{SMAD} 2 / 3$ signaling in pancreatic cancer cells, BxPC-3 and Panc- 1 cells were treated with $2 \mathrm{mM}$ metformin. Following $48 \mathrm{~h}$, total cell protein was extracted and subjected to western blot analysis. As anticipated, the protein expression levels of TGF- $\beta 1$ were decreased following metformin treatment. Additionally, the protein expression levels of $\mathrm{p}-\mathrm{Smad} 2 / 3$ were also decreased by metformin treatment in both BxPC-3 and Panc-1 cells (Fig. 2A). In addition, this phenomenon was confirmed by immunofluorescence assays (Fig. 2B). The results revealed that the expression levels of TGF- $\beta 1$ and $\mathrm{p}-\mathrm{Smad} 2 / 3$ were decreased by metformin treatment in pancreatic cancer cells. Notably, p-Smad2/3 was observed primarily in the cytoplasm following metformin treatment. In a subcutaneous tumor model, the immunofluorescence data revealed that TGF- $\beta 1$ staining in tumor tissue, as well as $\alpha$-SMA expression, was reduced by the oral administration of $200 \mathrm{mg} / \mathrm{kg}$ metformin (Fig. 2C). These results were consistent with those of our previous study (22). These findings revealed that metformin suppressed TGF- $\beta 1 / \mathrm{Smad} 2 / 3$ signaling in pancreatic cancer cells. 
A

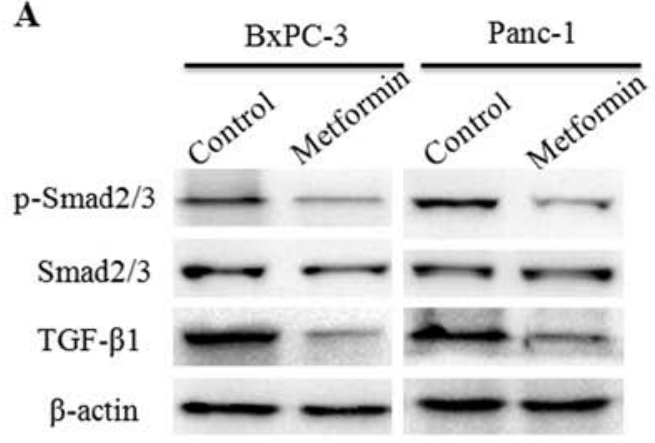

B

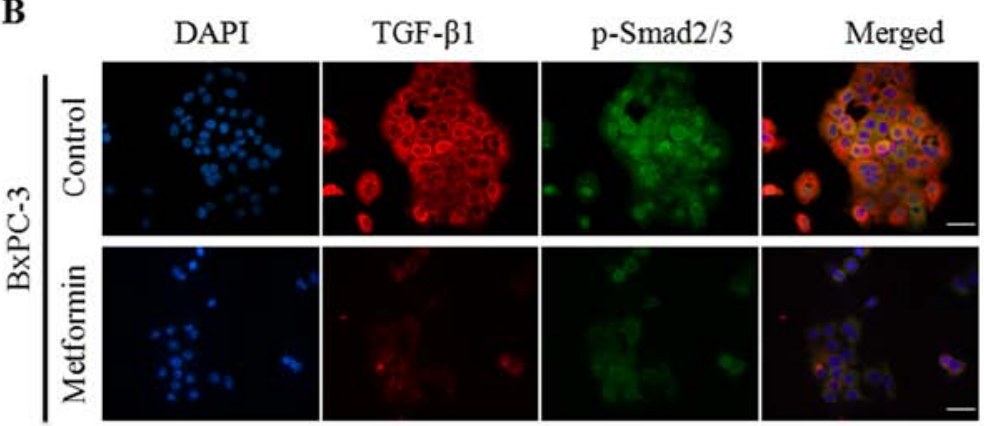

C
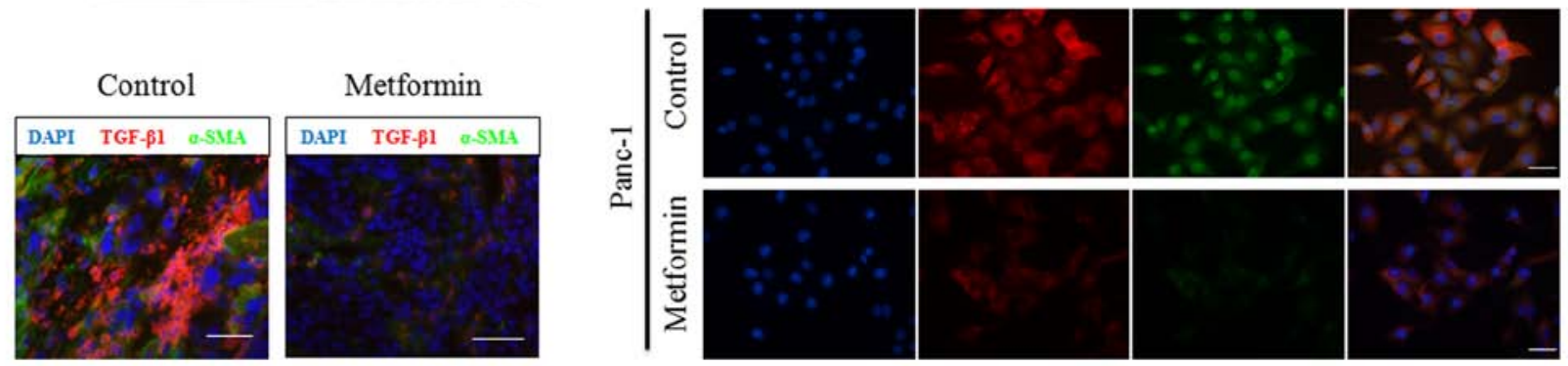

Figure 2. Metformin suppresses TGF- $\beta 1$ production and TGF- $\beta 1 / \mathrm{Smad} 2 / 3$ signaling in pancreatic cancer cells. (A) Panc- 1 and BxPC- 3 cells were treated with $2 \mathrm{mM}$ metformin for $48 \mathrm{~h}$ and then, total cell protein samples were prepared and analyzed by immunoblotting with antibodies against TGF- $\beta 1$, Smad $2 / 3$ and p-Smad2/3. (B) Immunofluorescence analyses were conducted to detect TGF- $\beta 1$ (red) and p-Smad2/3 (green) expression in Panc-1 and BxPC-3 cells following $2 \mathrm{mM}$ metformin treatment for $48 \mathrm{~h}$, magnification, x200. (C) Representative images displaying the immunofluorescence results of TGF- $\beta 1$ and $\alpha$-SMA staining of tumor tissue from a subcutaneous tumor model after the mice were orally administered $200 \mathrm{mg} / \mathrm{kg}$ metformin for 4 weeks. Scale bar, $50 \mu \mathrm{m}$.

$\mathbf{A}$

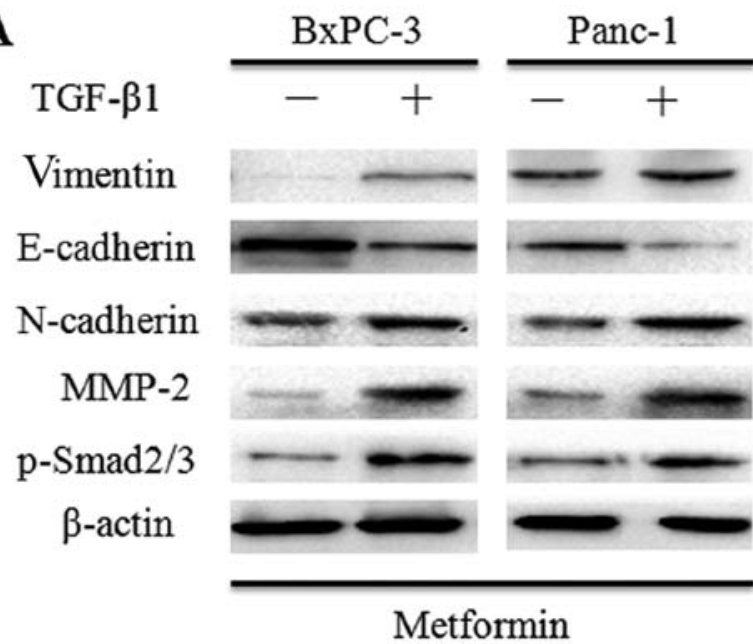

B
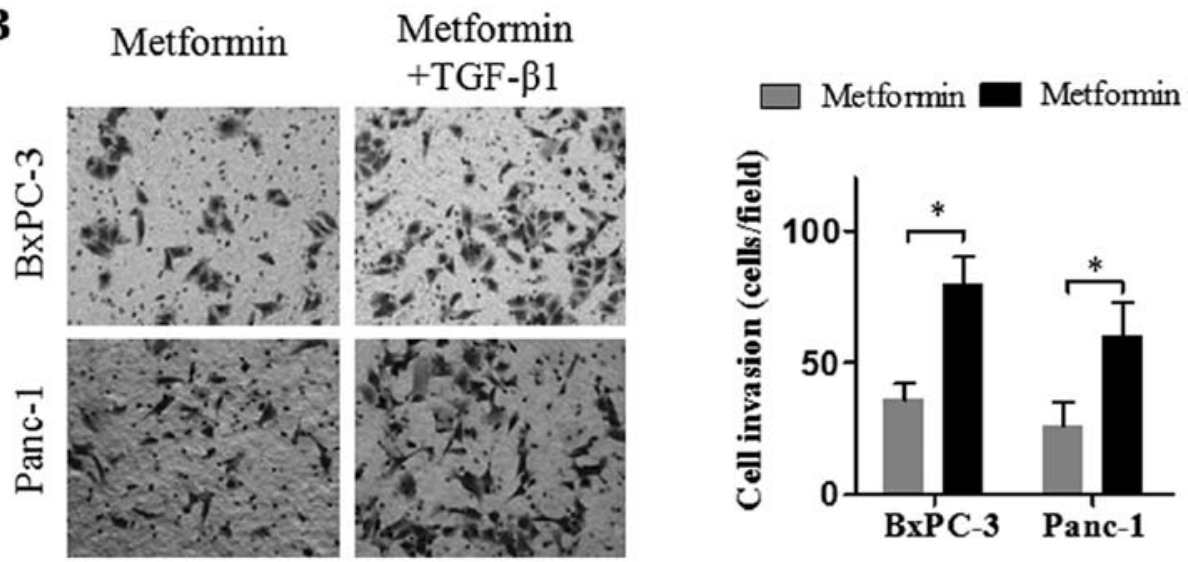

Figure 3. Recombinant TGF- $\beta 1$ reverses the metformin-induced EMT changes and invasion suppression in pancreatic cancer cells. (A) The expression levels of E-cadherin, N-cadherin, vimentin, MMP-2 and p-Smad2/3 were assessed by western blot assays after Panc- 1 and BxPC- 3 cells were treated with metformin $(2 \mathrm{mM})$ or metformin plus TGF- $\beta 1(2 \mathrm{ng} / \mathrm{ml})$ for $48 \mathrm{~h}$. (B) Matrigel invasion assays were performed to investigate the invasion ability of pancreatic cancer cells following treatment with metformin or metformin plus TGF- $\beta 1$ for $24 \mathrm{~h}$. Images are representative of three independent experiments. ${ }^{*} \mathrm{P}<0.05$, magnification, $\mathrm{x} 200$. 
A

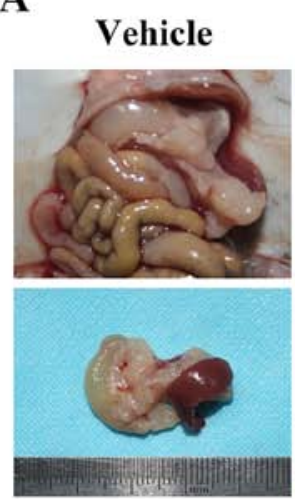

B

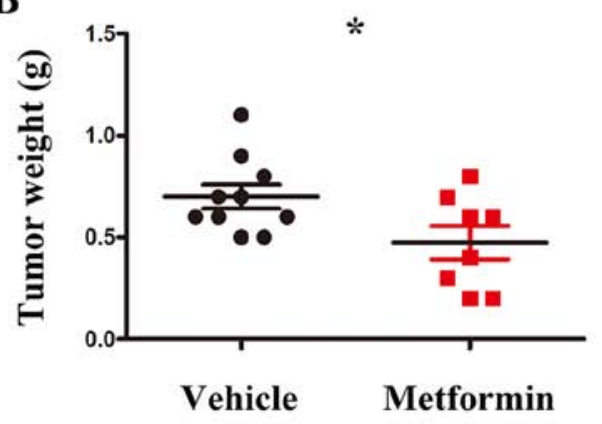

C
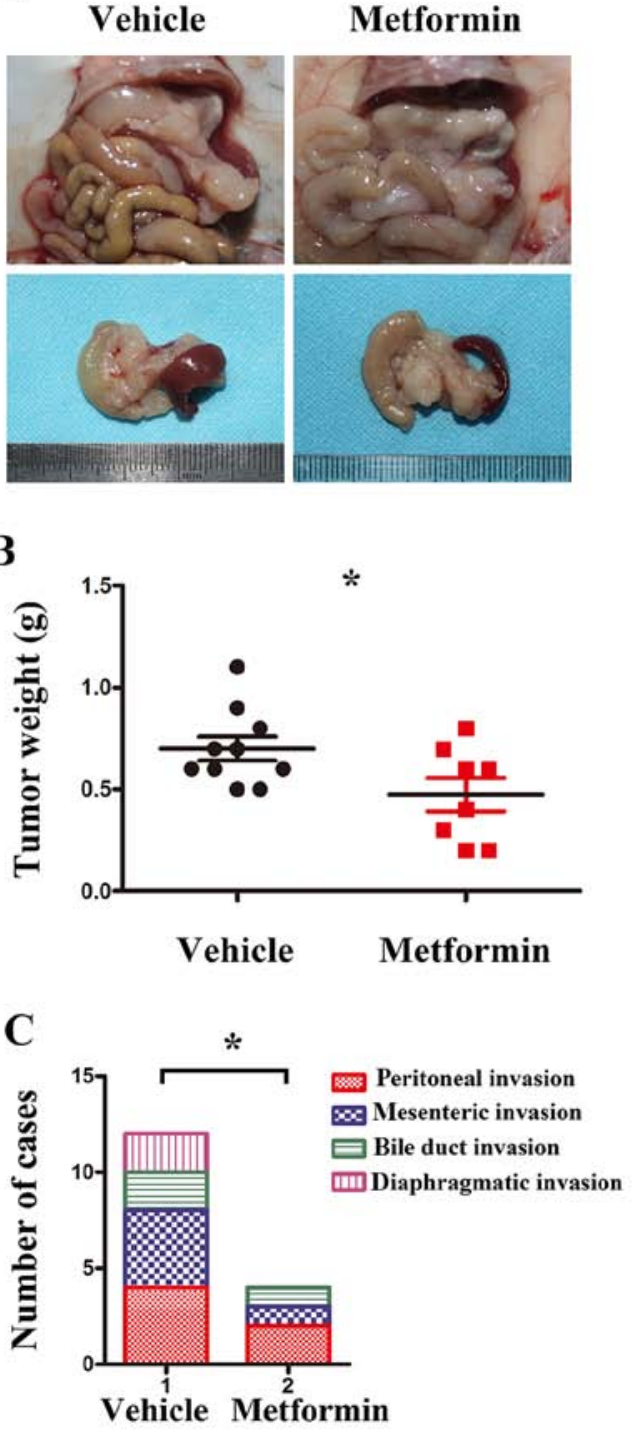

D
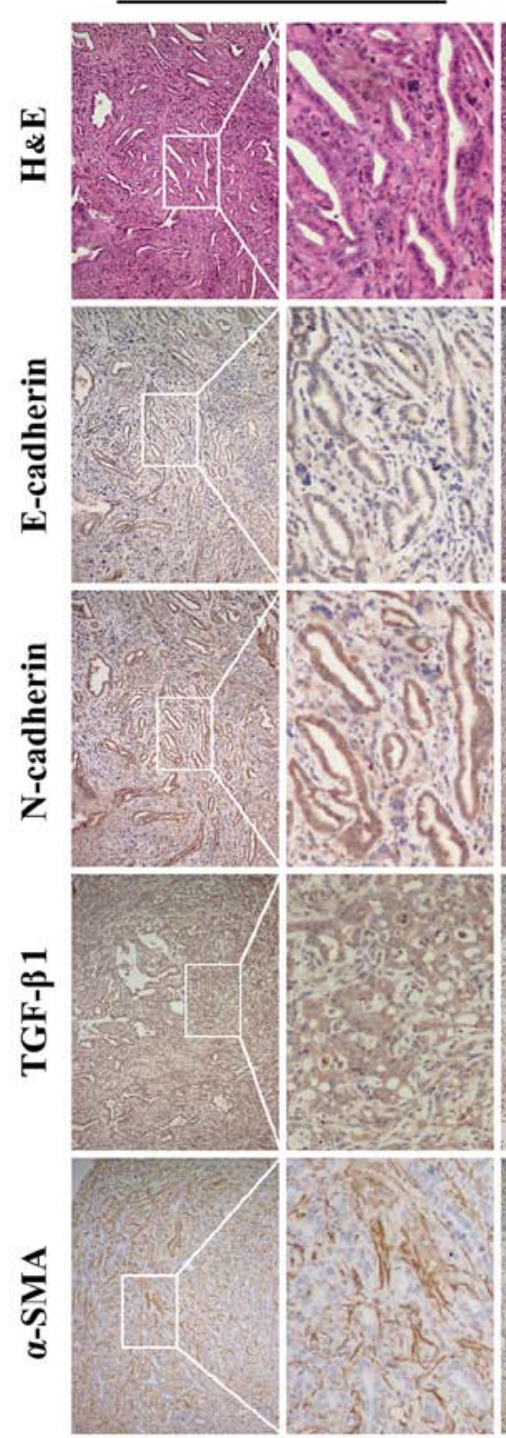
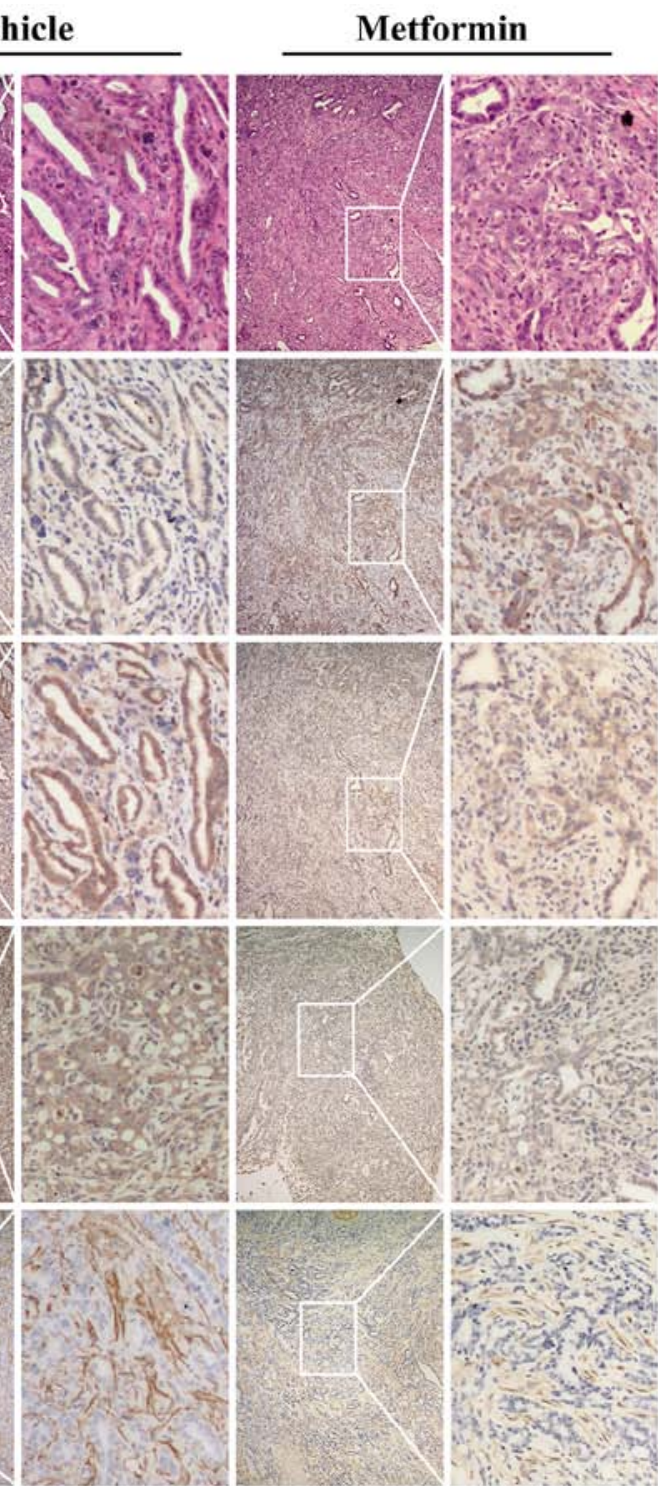

Figure 4. Metformin suppresses the growth, invasion and EMT of pancreatic cancer in a KPC transgenic mouse model. (A) Representative images displaying the macroscopic pancreatic tissue specimens from KPC mice treated with vehicle or metformin. (B) Tumor weights were quantified in mice treated with vehicle or metformin at the end of the experiment; ${ }^{*} \mathrm{P}<0.05$. (C) The occurrence of abdominal invasion (peritoneal invasion, mesenteric invasion, bile duct invasion and diaphragmatic invasion) was analyzed in KPC mice treated with vehicle or metformin; ${ }^{*} \mathrm{P}<0.05$. (D) Representative images of hematoxylin and eosin (H\&E) staining and immunohistochemical staining for E-cadherin, N-cadherin, TGF- $\beta 1$ and $\alpha$-SMA in tumor tissues from KPC mice treated with vehicle or metformin.

Exogenous TGF- $\beta 1$ reverses the effects of metformin on the invasion ability of pancreatic cancer cells. The aforementioned observations revealed that metformin suppressed the invasion ability and TGF- $\beta 1 / \mathrm{Smad} 2 / 3$ signaling in pancreatic cancer cells. To ascertain that TGF- $\beta 1$ signaling inhibition attenuated cancer cell invasion, Panc-1 and BxPC-3 cells were treated with metformin $(2 \mathrm{mM})$ alone or metformin plus recombinant TGF- $\beta 1(2 \mathrm{ng} / \mathrm{ml})$ for $24 \mathrm{~h}$ and then, EMT- and invasion-related markerswere detected by westernblotassays. The resultsrevealed that the addition of recombinant TGF- $\beta 1$ to the culture medium induced Smad2/3 phosphorylation (Fig. 3A). Furthermore, $\mathrm{N}$-cadherin, vimentin, and MMP-2 protein expression levels were increased, and E-cadherin protein expression levels were decreased in Panc-1 and BxPC-3 cells treated with metformin plus TGF- $\beta 1$, compared to cells treated with metformin alone. Matrigel invasion assays revealed that the metformin-mediated suppression of cancer cell invasion was almost recovered in the presence of TGF- $\beta 1$. Collectively, these observations indicated that TGF- $\beta 1$ downregulation may be responsible for the metformin-mediated invasion and EMT changes in pancreatic cancer cells.

Metformin suppresses pancreatic cancer tumor growth and metastasis in KPC transgenic mice. The KPC model is a well-validated, clinically relevant model of PDAC that recapitulates the spectrum of pancreatic cancer from pancreatic intraepithelial neoplasia (PanIN) to invasive PDAC (21). To determine the effects of metformin in vivo, KPC transgenic mice were generated and treated orally with vehicle (sterile water, daily for 4 weeks) or metformin $(200 \mathrm{mg} / \mathrm{kg}$, daily for 4 weeks). At the end of the experiment, KPC mice were sacrificed, and the tumor samples were prepared (Fig. 4A). 
The results revealed that the average tumor weight in the metformin-treated group was lower than that in the vehicle-treated group (Fig. 4B). In addition, compared with vehicle treatment, metformin treatment significantly decreased the incidence of abdominal invasion, including peritoneal invasion, mesenteric invasion and bile duct invasion as well as diaphragmatic invasion (Fig. 4C). The immunohistochemistry results (Fig. 4D) revealed that the TGF- $\beta 1$ immunoreactivity in the metformin-treated group was lower than that in the vehicle-treated group. In addition, increased E-cadherin and decreased $\mathrm{N}$-cadherin expression levels were observed in the metformin-treated group. Furthermore, compared with vehicle treatment, metformin treatment reduced the area of $\alpha$-SMA-positive staining. Collectively, these results indicated that metformin inhibited pancreatic cancer tumor growth, EMT and metastasis in vivo.

\section{Discussion}

PDAC is typically associated with a poor prognosis even after curative resection with postoperative adjuvant chemotherapy. Despite advances in our understanding of the molecular and genetic mechanisms of pancreatic cancer, managing the disease remains a clinical challenge due to its poor response to most chemotherapeutic agents (25). Local recurrence and metastasis are the primary causes of treatment failure in cancer patients and of cancer-related deaths. Recently, mounting evidence from both observational and laboratory studies indicated that metformin treatment may be associated with a decreased risk of developing cancer and a better response to chemotherapy $(12,26)$. The potential mechanisms underlying the antitumor properties of metformin remain unclear. Our previous studies have indicated that metformin can block the interaction between cancer cells and PSCs by reducing cancer cell-derived cytokines (22). In the present study, we found that metformin treatment significantly attenuated the migration and invasion properties of pancreatic cancer cells. In addition, metformin suppressed EMT in pancreatic cancer cells. This outcome was also produced in KPC transgenic mice. Furthermore, our data revealed that these effects of metformin occurred in part via the downregulation of TGF- $\beta 1 / \mathrm{Smad} 2 / 3$ signaling in pancreatic cancer.

TGF- $\beta 1$ is a potent cytokine with marked functionalities, including the regulation of cell apoptosis, proliferation, differentiation and extracellular matrix production (27). TGF- $\beta 1$ binding to its receptor results in the phosphorylation and activation of the transcription factors Smad2/3. Once activated, Smad2/3 translocates into the nucleus to govern gene transcription. The role of TGF- $\beta 1$ during cancer initiation and progression is complex and paradoxical. TGF- $\beta 1$ functions as a tumor suppressor in normal and early-stage cancers by inducing cell-cycle arrest and apoptotic reactions and as a tumor promoter in late-stage cancers by promoting cancer growth, invasion and metastasis $(28,29)$. Parallel studies have suggested that TGF- $\beta 1$ is a potent inducer of EMT $(11,30)$. Adding TGF- $\beta 1$ to epithelial cells in culture is a convenient way to induce EMT in various epithelial cells (31). During EMT induction, TGF- $\beta 1$ rapidly activates PI $3 \mathrm{~K}, \mathrm{Akt}, \mathrm{mTOR}$ complex 1 (mTORC1) and S6 kinase, leading to increases in protein synthesis, cell motility and invasion (32). A previous study has indicated that TGF- $\beta 1$ acted in an autocrine manner to enhance tumor cell invasion in pancreatic cancer by upregulating MMP-2 (33). In another study, a significant association was found between the expression of TGF- $\beta 1$ and lymph node involvement and the depth of invasion in pancreatic cancer (34). In the present study, we found that TGF- $\beta 1$ expression in pancreatic cancer cells was decreased by metformin. Notably, metformin treatment inhibited TGF- $\beta 1$-induced Smad2/3 phosphorylation in pancreatic cancer cells.

The desmoplastic reaction, which is a result of the proliferation of activated PSCs and the increased deposition of extracellular matrix (ECM) components, is a prominent pathological characteristic of pancreatic cancer (35). Accumulating evidence has indicated that the desmoplastic reaction in pancreatic cancer contributes to the aggressive nature of this malignancy by fostering tumor growth and metastatic spread, as well as enhancing chemoradiotherapy resistance (36-39). Thus, strategies targeting cancer-stroma interactions may serve as a potential approach for pancreatic cancer treatment (8). In pancreatic cancer, PSCs are activated by tumor-stromal interactions, including direct contact with pancreatic cancer cells and paracrine growth factors, such as TGF- $\beta 1$, secreted from pancreatic cancer cells $(40,41)$. It has recently been reported that metformin reduces desmoplasia in pancreatic cancer by reducing the expression of inflammatory cytokines, resulting in reduced disease progression (42). Our previous study also indicated that metformin suppressed desmoplasia in pancreatic cancer by reducing TGF- $\beta 1$ production in pancreatic cancer cells and inhibiting paracrine-mediated PSC activation both in vitro and in vivo (22). The anti-stromal behavior of metformin has been previously verified in a genetically engineered mouse model of pancreatic cancer (21). In the present study, we also observed that $\alpha$-SMA-positive cells were markedly reduced in cancer tissues from KPC mice following metformin treatment. Collectively, this evidence indicated the anti-stromal properties of metformin, and that reduced TGF- $\beta 1$ production by cancer cells may be the underlying mechanism.

In the past, we conducted a series of studies on the anticancer effect of metformin on pancreatic cancer. Our previous study revealed that metformin suppressed desmoplasic reaction in pancreatic cancer by reducing TGF- $\beta 1$ production in cancer cells and suppressing paracrine TGF- $\beta 1$-induced PSC activation (22). In addition, both our in vitro and in vivo experiments revealed that metformin inhibited the proliferation, invasion and migration of pancreatic cancer cells $(22,43)$. Furthermore, by using a genetic mouse model of pancreatic cancer, our results confirmed the inhibitory effect of metformin on pancreatic cancer initiation and progression (21). However, whether the inhibitory effect of metformin on tumor invasion and metastasis is mediated by targeting the autocrine TGF- $\beta 1 /$ Smad pathway remains unknown. Based on the aforementioned research, in the present study, we investigated the effect of metformin on TGF- $\beta 1 /$ Smad in pancreatic cancer cells. Our results indicated that blocking autocrine TGF- $\beta 1$ signaling was partially responsible for metformin-reduced cell invasion properties. Therefore, the present study is a confirmation and supplement to our previous studies.

In conclusion, the results from the present study indicated that metformin suppressed the invasion, EMT and metastasis 
of pancreatic cancer by preventing autocrine TGF- $\beta 1 / \mathrm{Smad} 2 / 3$ signaling both in vitro and in vivo. Thus, our study revealed a new possible mechanism for the antitumor effects of metformin.

\section{Acknowledgements}

Not applicable.

\section{Funding}

The present study was supported by grants from the National Natural Science Foundation of China (nos. 81402971, 81672434 and 81702916).

\section{Availability of data and materials}

The datasets used during the present study are available from the corresponding author upon reasonable request.

\section{Authors' contributions}

QM and WD designed the experiments. WQ, CZ and JC performed the majority of the experiments. TQ, YX, LC and JL analyzed the data. KC and XL organized the figures. JM wrote the manuscript. KC, XL and JM were also involved in the conception of the study. QM and WD reviewed it. All authors read and approved the manuscript and agree to be accountable for all aspects of the research in ensuring that the accuracy or integrity of any part of the work are appropriately investigated and resolved.

\section{Ethics approval and consent to participate}

All experimental protocols were authorized by the Ethics Committee of the First Affiliated Hospital of Medical College, Xi'an Jiaotong University (Xi'an, China). The protocols also complied with the Declaration of Helsinki.

\section{Patient consent for publication}

Not applicable.

\section{Competing interests}

The authors declare that they have no competing interests.

\section{References}

1. Siegel RL, Miller KD and Jemal A: Cancer statistics, 2016. CA Cancer J Clin 66: 7-30, 2016.

2. Kamisawa T, Wood LD, Itoi T and Takaori K: Pancreatic cancer. Lancet 388: 73-85, 2016.

3. Chiu J and Yau T: Metastatic pancreatic cancer: Are we making progress in treatment? Gastroenterol Res Pract 2012: 898931, 2012.

4. Hanahan D and Weinberg RA: Hallmarks of cancer: The next generation. Cell 144: 646-674, 2011.

5. Micalizzi DS, Haber DA and Maheswaran S: Cancer metastasis through the prism of epithelial-to-mesenchymal transition in circulating tumor cells. Mol Oncol 11: 770-780, 2017.

6. Morandi A, Taddei ML, Chiarugi P and Giannoni E: Targeting the metabolic reprogramming that controls epithelial-tomesenchymal transition in aggressive tumors. Front Oncol 7: 40, 2017.
7. Yilmaz M and Christofori G: EMT, the cytoskeleton, and cancer cell invasion. Cancer Metastasis Rev 28: 15-33, 2009.

8. Maeda M, Johnson KR and Wheelock MJ: Cadherin switching: Essential for behavioral but not morphological changes during an epithelium-to-mesenchyme transition. J Cell Sci 118: 873-887, 2005.

9. Wu Q, Miele L, Sarkar FH and Wang Z: The role of EMT in pancreatic cancer progression. Pancreat Disord Ther 2: pii: e121, 2012.

10. Rhim AD,MirekET,AielloNM,Maitra A,Bailey JM,McAllisterF, Reichert M, Beatty GL, Rustgi AK, Vonderheide RH, et al: EMT and dissemination precede pancreatic tumor formation. Cell 148: 349-361, 2012.

11. Lamouille S, Connolly E, Smyth JW, Akhurst RJ and Derynck R: TGF- $\beta$-induced activation of mTOR complex 2 drives epithelial-mesenchymal transition and cell invasion. J Cell Sci 125: 1259-1273, 2012.

12. Decensi A, Puntoni M, Goodwin P, Cazzaniga M, Gennari A, Bonanni B and Gandini S: Metformin and cancer risk in diabetic patients: A systematic review and meta-analysis. Cancer Prev Res 3: 1451-1461, 2010.

13. Noto H, Goto A, Tsujimoto T and Noda M: Cancer risk in diabetic patients treated with metformin: A systematic review and meta-analysis. PLoS One 7: e33411, 2012.

14. Wright JL and Stanford JL: Metformin use and prostate cancer in Caucasian men: Results from a population-based case-control study. Cancer Causes Control 20: 1617-1622, 2009.

15. Currie CJ, Poole CD and Gale EA: The influence of glucose-lowering therapies on cancer risk in type 2 diabetes. Diabetologia 52: 1766-1777, 2009.

16. Li D, Yeung SC, Hassan MM, Konopleva M and Abbruzzese JL: Antidiabetic therapies affect risk of pancreatic cancer. Gastroenterology 137: 482-488, 2009.

17. Aljada A and Mousa SA: Metformin and neoplasia: Implications and indications. Pharmacol Ther 133: 108-115, 2012.

18. Bhalla K, Hwang BJ, Dewi RE, Twaddel W, Goloubeva OG, Wong KK, Saxena NK, Biswal S and Girnun GD: Metformin prevents liver tumorigenesis by inhibiting pathways driving hepatic lipogenesis. Cancer Prev Res 5: 544-552, 2012.

19. Kato K, Gong J, Iwama H, Kitanaka A, Tani J, Miyoshi H, Nomura K, Mimura S, Kobayashi M, Aritomo Y, et al: The antidiabetic drug metformin inhibits gastric cancer cell proliferation in vitro and in vivo. Mol Cancer Ther 11: 549-560, 2012.

20. Courtois S, Durán RV, Giraud J, Sifré E, Izotte J, Mégraud F, Lehours $\mathrm{P}$, Varon $\mathrm{C}$ and Bessède $\mathrm{E}$ : Metformin targets gastric cancer stem cells. Eur J Cancer 84: 193-201, 2017.

21. Chen K, Qian W, Jiang Z, Cheng L, Li J, Sun L, Zhou C, Gao L, Lei M, Yan B, et al: Metformin suppresses cancer initiation and progression in genetic mouse models of pancreatic cancer. Mol Cancer 16: 131, 2017.

22. Duan W, Chen K, Jiang Z, Chen X, Sun L, Li J, Lei J, Xu Q, Ma J, $\mathrm{Li}$ X, et al: Desmoplasia suppression by metformin-mediated AMPK activation inhibits pancreatic cancer progression. Cancer Lett 385: 225-233, 2017

23. Duan W, Li R, Ma J, Lei J, Xu Q, Jiang Z, Nan L, Li X, Wang Z, Huo $\mathrm{X}$, et al: Overexpression of Nodal induces a metastatic phenotype in pancreatic cancer cells via the $\operatorname{Smad} 2 / 3$ pathway. Oncotarget 6: 1490-1506, 2015.

24. Jiang Z, Chen X, Chen K, Sun L, Gao L, Zhou C, Lei M, Duan W, Wang Z, Ma Q and Ma J: YAP inhibition by resveratrol via activation of AMPK enhances the sensitivity of pancreatic cancer cells to gemcitabine. Nutrients 8: pii: E546, 2016.

25. Collisson EA and Olive KP: Pancreatic cancer: Progress and challenges in a rapidly moving field. Cancer Res 77: 1060-1062, 2017.

26. Zhang HH and Guo XL: Combinational strategies of metformin and chemotherapy in cancers. Cancer Chemother Pharmacol 78: 13-26, 2016.

27. Tian M, Neil JR and Schiemann WP: Transforming growth factor- $\beta$ and the hallmarks of cancer. Cell Signal 23: 951-962, 2011.

28. Morrison CD, Parvani JG and Schiemann WP: The relevance of the TGF- $\beta$ Paradox to EMT-MET programs. Cancer Lett 341 : 30-40, 2013.

29. Ikushima $\mathrm{H}$ and Miyazono K: TGFbeta signalling: A complex web in cancer progression. Nat Rev Cancer 10: 415-424, 2010.

30. Pirozzi G, Tirino V, Camerlingo R, Franco R, La Rocca A, Liguori E, Martucci N, Paino F, Normanno N and Rocco G: Epithelial to mesenchymal transition by TGF $\beta-1$ induction increases stemness characteristics in primary non small cell lung cancer cell line. PLoS One 6: e21548, 2011. 
31. Xu J, Lamouille S and Derynck R: TGF-beta-induced epithelial to mesenchymal transition. Cell Res 19: 156-172, 2009.

32. Lamouille S and Derynck R: Cell size and invasion in TGF-beta-induced epithelial to mesenchymal transition is regulated by activation of the mTOR pathway. J Cell Biol 178: 437-451, 2007.

33. Ellenrieder V, Hendler SF, Ruhland C, Boeck W, Adler G and Gress TM: TGF-beta-induced invasiveness of pancreatic cancer cells is mediated by matrix metalloproteinase- 2 and the urokinase plasminogen activator system. Int J Cancer 93: 204-211, 2001.

34. Yin T, Wang C, Liu T, Zhao G and Zhou F: Implication of EMT induced by TGF-beta1 in pancreatic cancer. J Huazhong Univ Sci Technol Med Sci 26: 700-702, 2006.

35. Feig C, Gopinathan A, Neesse A, Chan DS, Cook N and Tuveson DA: The pancreas cancer microenvironment. Clin Cancer Res 18: 4266-4276, 2012.

36. Apte MV, Park S, Phillips PA, Santucci N, Goldstein D, Kumar RK, Ramm GA, Buchler M, Friess H, McCarroll JA, et al: Desmoplastic reaction in pancreatic cancer: Role of pancreatic stellate cells. Pancreas 29: 179-187, 2004.

37. Tang D, Wang D, Yuan Z, Xue X, Zhang Y, An Y, Chen J, Tu M, Lu Z, Wei J, et al: Persistent activation of pancreatic stellate cells creates a microenvironment favorable for the malignan behavior of pancreatic ductal adenocarcinoma. Int J Cancer 132: 993-1003, 2013.

38. Lonardo E, Frias-Aldeguer J, Hermann PC and Heeschen C: Pancreatic stellate cells form a niche for cancer stem cells and promote their self-renewal and invasiveness. Cell Cycle 11: 1282-1290, 2012.
39. Mantoni TS, Lunardi S, Al-Assar O, Masamune A and Brunner TB: Pancreatic stellate cells radioprotect pancreatic cancer cells through $\beta 1$-integrin signaling. Cancer Res 71: 3453-3458, 2011

40. Löhr M, Schmidt C, Ringel J, Kluth M, Müller P, Nizze H and Jesnowski R: Transforming growth factor-betal induces desmoplasia in an experimental model of human pancreatic carcinoma. Cancer Res 61: 550-555, 2001.

41. Vonlaufen A, Phillips PA, Xu Z, Goldstein D, Pirola RC, Wilson JS and Apte MV: Pancreatic stellate cells and pancreatic cancer cells: An unholy alliance. Cancer Res 68: 7707-7710, 2008.

42. Incio J, Suboj P, Chin SM, Vardam-Kaur T, Liu H, Hato T, Babykutty S, Chen I, Deshpande V, Jain RK and Fukumura D: Metformin reduces desmoplasia in pancreatic cancer by reprogramming stellate cells and tumor-associated macrophages. PLoS One 10: $\mathrm{e} 0141392,2015$.

43. Chen K, Qian W, Li J, Jiang Z, Cheng L, Yan B, Cao J, Sun L, Zhou C, Lei M, et al: Loss of AMPK activation promotes the invasion and metastasis of pancreatic cancer through an HSF1-dependent pathway. Mol Oncol 11: 1475-1492, 2017. 